\title{
EFFECT OF GERANYLGERANYLACETONE ON ASPIRIN-INDUCED CHANGES IN GASTRIC GLYCOPROTEINS
}

\author{
Kiyoshi OKETANI, Manabu MURAKAMI, Hideaki FUJISAKI \\ and Tsuneo WAKABAYASHI \\ Department of Pharmacology. Tsukuba Research Laboratories, \\ Eisai Co., Ltd., Ibaragi 300-26, Japan \\ Kyoko HOTTA \\ Department of Biochemistry. Kitasato University School of Medicine. \\ Kanagawa 228, Japan
}

Accepted January 20, 1983

\begin{abstract}
The effects of aspirin and treatment with geranylgeranylacetone (GGA), an antiulcer drug, on the content of gastric glycoproteins were investigated in pylorusligated rats. In normal rats, the amount of gastric glycoproteins in the mucous layer was about 1.5 times higher than that in the gastric mucosa, indicating that the glycoproteins were distributed in the mucous layer as a highly concentrated state. Aspirin (100 mg/kg. p.o.) decreased the content of gastric glycoproteins both in the mucous layer and in the gastric mucosa. The amount of the macromolecular fraction in the gastric juice, which corresponded to the gastric glycoproteins on the basis of molecular size, was not affected by aspirin. GGA (300 $\mathrm{mg} / \mathrm{kg}$. i.d.) could prevent the decreases of the total amount of gastric glycoproteins in the mucous layer plus gastric mucosa. These results indicated that the glycoproteins coating the surface of the gastric mucosa may play a role as a defensive mechanism and that GGA exerted an antiulcer effect on aspirininduced mucosal damage through preventing the decreases in gastric glycoproteins.
\end{abstract}

The inhibitory effect of aspirin on the biosynthesis of glycoproteins in the gastric mucosa has been reported, i.e., decreases in ${ }^{35} \mathrm{SO}_{4}{ }^{2-}$ incorporation into the sulfated glycoproteins (1) and changes of carbohydrate content of the glycoproteins (2). Recently. Azuumi et al. (3) isolated the macromolecular glycoproteins from gastric mucosa using gel filtration, and they found that only macromolecular glycoproteins were decreased after administration of aspirin.

These results have led to the hypothesis that aspirin may cause gastric mucosal injury by inhibiting the synthesis of glycoproteins, but the precise location and role of the glycoproteins have not been fully defined.
The present study was undertaken to investigate the changes in gastric glycoproteins in the mucous layer and the gastric mucosa and in the gastric juice induced by aspirin. Additionally, the effect of geranylgeranylacetone (GGA). which had an antiulcer potency against aspirin-induced lesions (4), on the changes of the glycoproteins was examined.

\section{Materials and Methods}

Male Sprague-Dawley rats, weighing about $170 \mathrm{~g}$. were used in these experiments. Animals were housed in a room maintained at a temperature of $22 \pm 2^{\circ} \mathrm{C}$ and at a humidity of $55 \pm 5 \%$. They were deprived of food for $24 \mathrm{hr}$ before the experiments, but had free 
access to water.

Geranylgeranylacetone (all-trans-geranylgeranylacetone: 5-cis, 9-trans, 13-transgeranylgeranylacetone $=6: 4 ;$ GGA) was synthesized at the Eisai Co., Ltd. and emulsified in $5 \%$ gum arabic and $0.6 \%$ Tween 80 .

Aspirin ulcer in pylorus-ligated rats: According to the method of Okabe et al. (5), the pylorus of each animal was ligated under light ether anesthesia. Immediately after the ligation, aspirin was administered orally at a dose of $100 \mathrm{mg} / \mathrm{kg}$, and the test drug was given intraduodenally at a dose of $300 \mathrm{mg} /$ $\mathrm{kg}$. Seven hr after the ligation, the animals were sacrificed under ether anesthesia. The stomach was removed, and the gastric juice was collected.

Analysis of the gastric glycoproteins by gel filtration: The amount of gastric glycoproteins in the mucous layer and the gastric mucosa were determined as previously reported (6) with gel filtration method (3). The stomach was incised along the greater curvature after removing the forestomach. From one half of the stomach, the mucous layer was scraped off with gauze, and the other half was left untreated. After lyophilizing. each stomach half was homogenized in $6 \mathrm{ml}$ of $2 \%$ Triton $X-100 / 50 \mathrm{mM}$ Tris $-\mathrm{HCl}$. $\mathrm{pH} 7.2$, with an Ultra Turax (Janke Kunkel KG. Type TP 18/10 S2) and centrifuged at $10,000 \mathrm{rpm}$ for $30 \mathrm{~min}$. One $\mathrm{ml}$ of the supernatant was applied on a column of BioGel A 1.5 m (100-200 mesh. Biorad Laboratories, $1 \times 35 \mathrm{~cm}$ ), which had been equilibrated with the same buffer, and eluted at room temperature.

Fractions of $1 \mathrm{ml}$ were collected (Toyo. SF $160 \mathrm{~K}$ ), and aliquots were taken for the measurement of hexose by the phenol-sulfric acid method (7) and protein by the method of Wang and Smith (8). Hexosamine was determined in pooled fractions according to the method of Neuhaus and Letzring (9).

The content of the glycoproteins in the mucous layer and gastric mucosa were determined as follows:

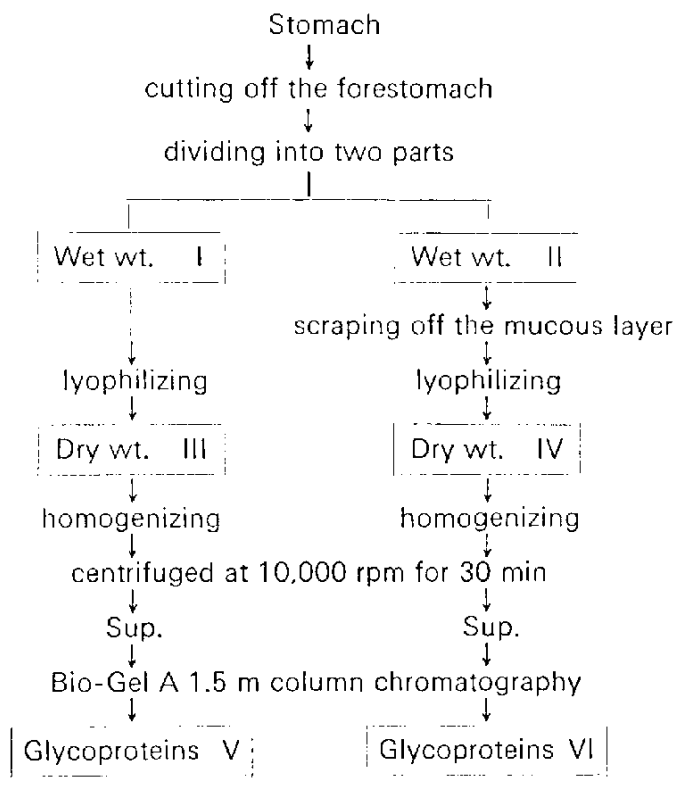

The content of the gastric glycoproteins was expressed as $\mathrm{mg}$ of hexose per $\mathrm{mg}$ of dry tissue (concentration) and $\mu g$ of hexose per one stomach (total amount).

$$
\begin{aligned}
& \text { Concentration of Glycoproteins ( } \mathrm{mg} / \mathrm{mg} \text { dry tissue) } \quad=\mathrm{V} / \mathrm{III} \\
& \text { Total amount of Glycoproteins ( } \left.\mu \mathrm{g} / \text { stomach }^{*}\right) \quad=V(I+\mid \mathrm{l}) / \mathrm{l}-(\mathrm{a}) \\
& \left(\mu \mathrm{g} / \text { gastric mucosa }{ }^{*}\right)=V((i+11) / 11-(b) \\
& \left(\boldsymbol{\mu} \mathrm{g} / \text { mucous layer }{ }^{*}\right) \quad=(\mathrm{a})-(\mathrm{b})
\end{aligned}
$$

*Total glycoproteins in the stomach=glycoproteins in the gastric mucosa plus mucous laver.

Collected gastric juice was centrifuged at $10.000 \mathrm{rpm}$ for $30 \mathrm{~min}$, and $1 \mathrm{ml}$ of the supernatant was applied on a column of Bio-Gel A $1.5 \mathrm{~m}$. The eluted fractions were analyzed for hexose and protein as described above.

Histochemical study of the stomach: The stomach opened along the greater curvature 
was fixed in $95 \%$ ethanol and embeded in paraffin. Four $u m$ paraffin sections were prepared and stained with periodic-acidSchiff (PAS).

The Student's t-test was used for statistical analysis.

\section{Results}

Changes in PAS positive substances in the stomach: As shown in Fig. 1. PAS positive substances were distributed in the superficial mucous layer and in the neck of the gland of the gastric mucosa. These substances in both layers were reduced after administration of aspirin at a dose of $100 \mathrm{mg} / \mathrm{kg}$ to pylorusligated rats. In aspirin plus GGA $(300 \mathrm{mg} /$ $\mathrm{kg}$ ) treated animals. PAS positive substances were maintained at the normal level

Effect of aspirin and treatment of GGA on the gastric glycoproteins: As shown in Fig. 2. the elution pattern of the extract of normal stomach showed three peaks of hexose (F-1. $\mathrm{F}-2$ and $\mathrm{F}-3$ ) which contained a protein component. Particularly in F-1, the peak of hexose and protein was found in the same position at the void volume. Hexose contents and hexosamine contents in $\mathrm{F}-1, \mathrm{~F}-2$ and $\mathrm{F}-3$ were $23 \%, 25 \%, 52 \%$ and $64 \%, 21 \%, 15 \%$. respectively (Fig. 3). The nonretarded fraction ( $F-1)$ with an elution position coinciding with that of protein, containing a high percentage of hexosamine. was designated as "gastric glycoproteins" in this paper.

Figure 4 showed the characteristic column elution profiles of the stomach of the normal rat, aspirin treated rat (Control), and aspirin plus GGA treated rat. The peak designated as gastric glycoproteins ( $F-1$ ) was significantly reduced by aspirin, while the other two peaks of lower molecular weight fractions (F-2. F-3) were not changed. In the aspirin plus GGA treated rat, the elution profile of the stomach was similar to that of the normal rat. The changes in hexose contents in $\mathrm{F}-1$.
(A)

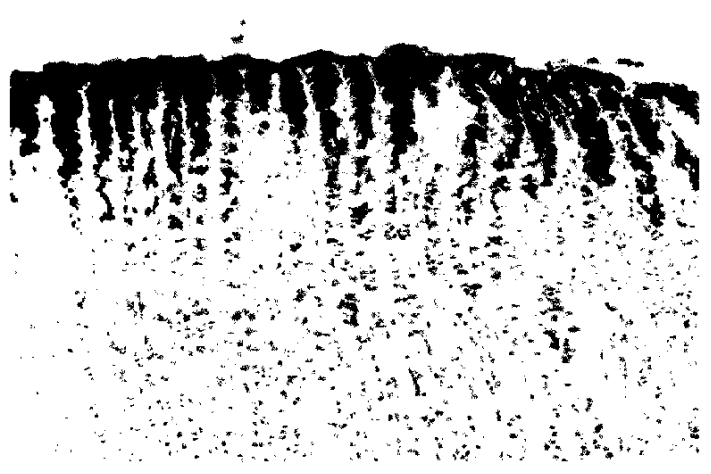

(B)

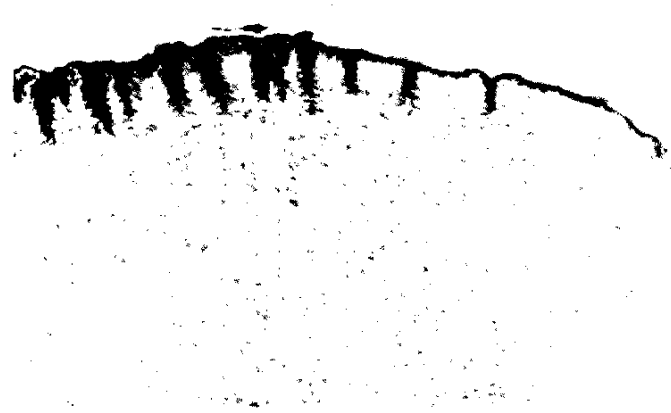

(C)

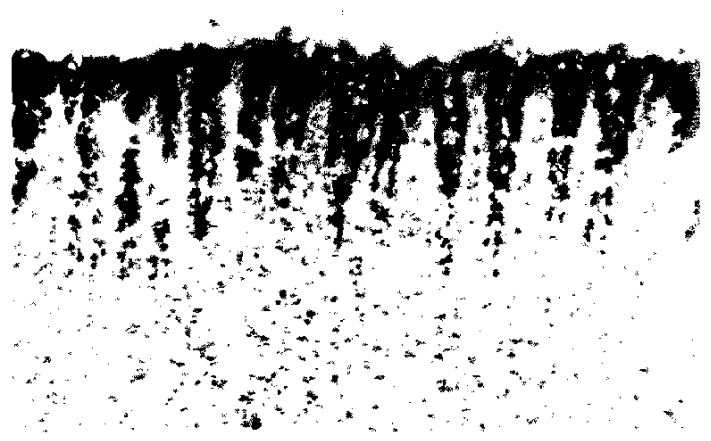

Fig. 1. Histochemical study of the stomach in rats. (A) Normal, (B) Control (Aspirin). (C) GGA (300 $\mathrm{mg} / \mathrm{kg}$ ).

$F-2$ and $F-3$ fractions of the stomach are summarized in Table 1: F-1, F-2 and F-3 fractions from the Bio-Gel A $1.5 \mathrm{~m}$ column were pooled as shown in Fig. 4, and hexose content in each fraction was determined. In the normal rat. the hexose contents in F-1, $\mathrm{F}-2$ and $\mathrm{F}-3$ were $9.22 \pm 0.15,7.60 \pm 0.50$, 


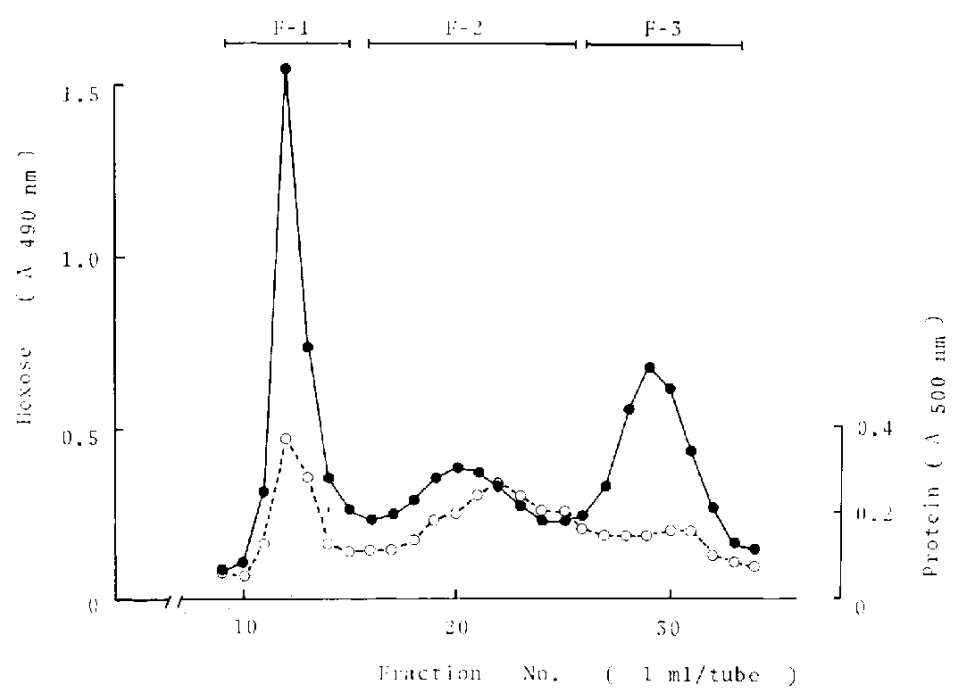

Fig. 2. Bio-Gel A $1.5 \mathrm{~m}$ column chromatography of the extract of the rat stomach.

Hexose, (O) Protein.

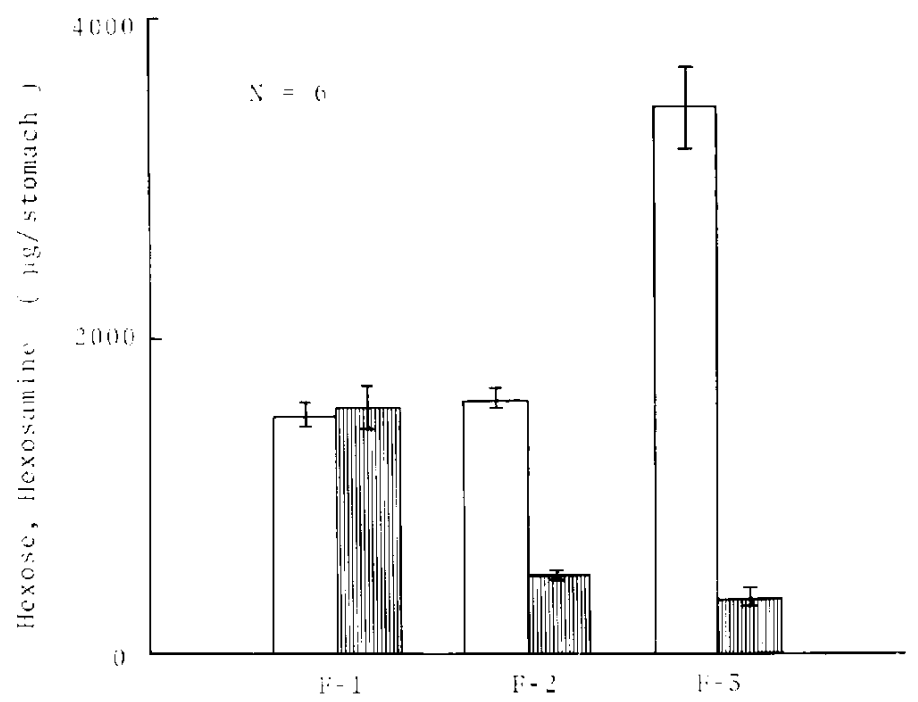

Fig. 3. Distribution of the amount of hexose and hexosamine in the fractions of the Bio-Gel A $1.5 \mathrm{~m}$ column from the rat stomach. $\square$. Hexose. | IIi $\mid$ Hexosamine.

$12.22 \pm 0.88 \mathrm{mg} / \mathrm{mg}$ of dry tissue, respectively. Oral administration of aspirin at $100 \mathrm{mg} / \mathrm{kg}$ to pylorus-ligated rats, which produced severe mucosal damage in the glandular portion, caused $18 \%$ decreases in the concentration of gastric glycoproteins $(F-1)$, significantly, but did not affect the lower molecular fractions (F-2, F-3). In aspirin plus GGA treated rats, the decreases in gastric glycoproteins ( $F-1$ ) were $7 \%$ of the normal level. These results suggest that GGA prevented the decreases in gastric glycoproteins ( $F-1$ ) contents induced by aspirin. The changes in the gastric glycoproteins (F-1) were investigated by separating the stomach into the "mucous layer" and the 

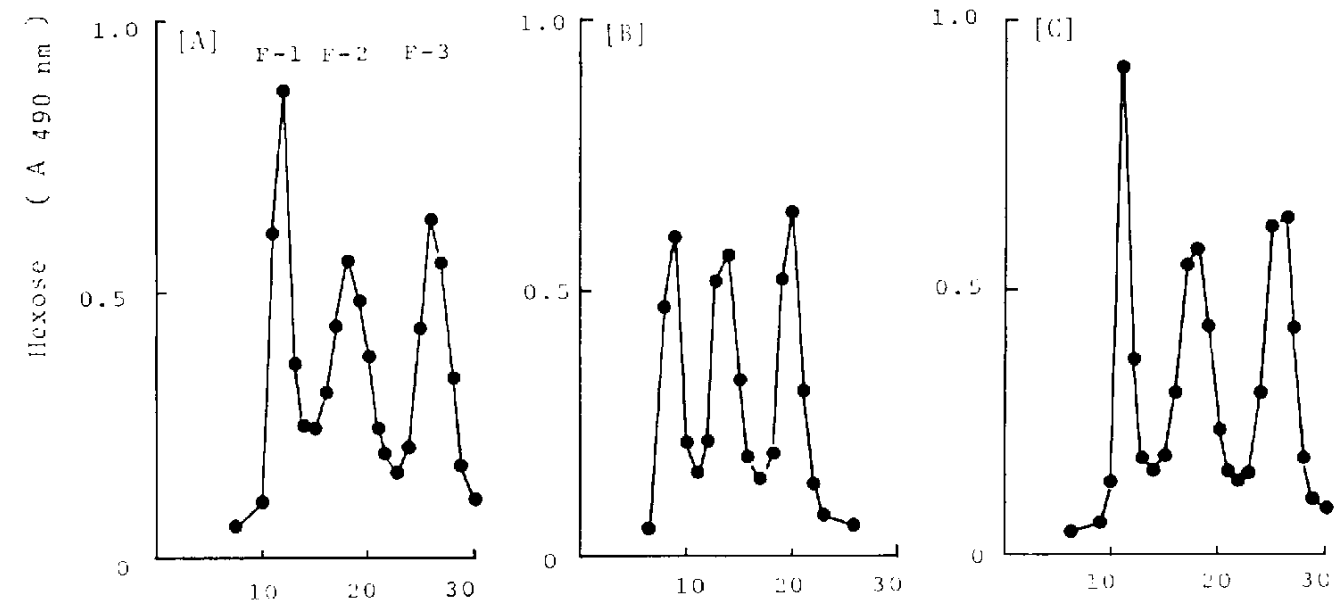

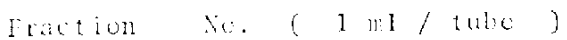

Fig. 4. Changes in elution pattern of the extract of stomach on the Bio-Gel A $1.5 \mathrm{~m}$ column. (A) Normal, (B) Control (Aspirin), (C) GGA (300 mg/kg).

Table 1 Effect of GGA on hexose content of Bio-Gel A $1.5 \mathrm{~m}$ fractions from the stomach in rats treated with aspirin

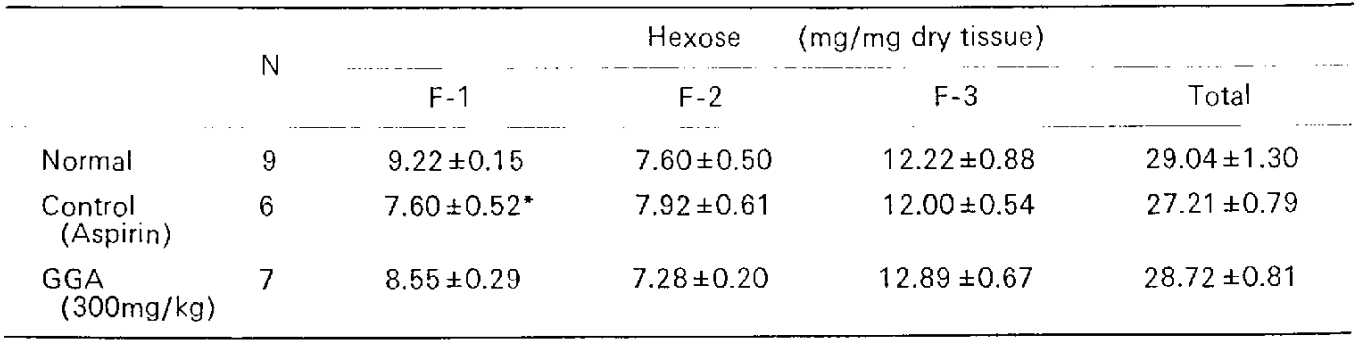

All values represent the mean \pm S.E. $\quad *<<0.05$ when compared with normal.

"gastric mucosa". In normal rats, total amounts of the gastric glycoproteins $(F-1)$ in the mucous layer, the gastric mucosa, and in the mucous layer plus gastric mucosa were $822 \pm 38,535 \pm 33$ and $1360 \pm 35 \mu \mathrm{g}$, respectively (Fig. 5), and the concentrations of gastric glycoproteins ( $F-1)$ in each layer were $22.1 \pm 0.6,4.9 \pm 0.3$ and $9.2 \pm 0.2 \mathrm{mg} /$ $\mathrm{mg}$ of dry tissue, respectively. Comparison of the content of gastric glycoproteins ( $F-1$ ) in the mucous layer with that in the gastric mucosa indicated that the gastric glycoproteins ( $F-1$ ) were distributed mainly in the mucous layer. As shown in Fig. 5, seven hr after the administration of aspirin, the amounts of gastric glycoproteins $(\mathrm{F}-1)$ in the mucous layer and gastric mucosa were significantly decreased by $23 \%$ and $32 \%$. respectively. In aspirin plus GGA treated rats, the decreases in the glycoproteins (F-1) in each layer were $9 \%$ and $13 \%$, respectively. The decreases in total gastric glycoproteins $(\mathrm{F}-1)$ in the mucous layer plus gastric mucosa were significantly prevented by GGA.

On the other hand, the lower molecular fractions (F-2, F-3) were predominantly distributed in the gastric mucosa $(80 \%$. $85 \%$ of the total amount, respectively) and were not affected by aspirin and GGA (data not shown). 
Effect of aspirin and GGA on the Bio- was separated into two size classes on the Gel A $1.5 \mathrm{~m}$ column fractions of gastric juice: basis of hexose content by Bio-Gel A $1.5 \mathrm{~m}$ The gastric juice collected from Shay rats column filtration (Fig. 6A). The larger

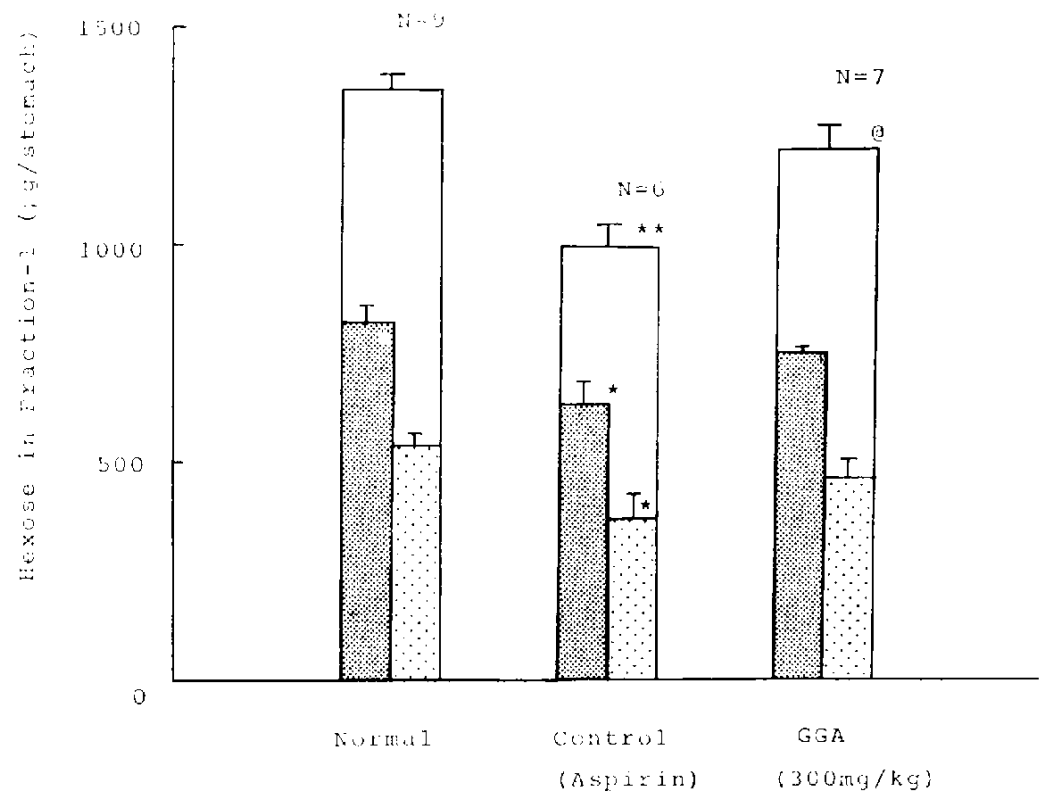

Fig. 5. Effect of GGA on aspirin-induced changes in the amount of gastric glycoproteins in the mucous layer and gastric mucosa. 1 Mucous layer, $;:$ Gastric mucosa. $\square$ Mucous layer+Gastric mucosa. "P<0.05, " $\mathrm{P}<0.01$ vs. Normal. $Q \mathrm{P}<0.05$ vs. Control.
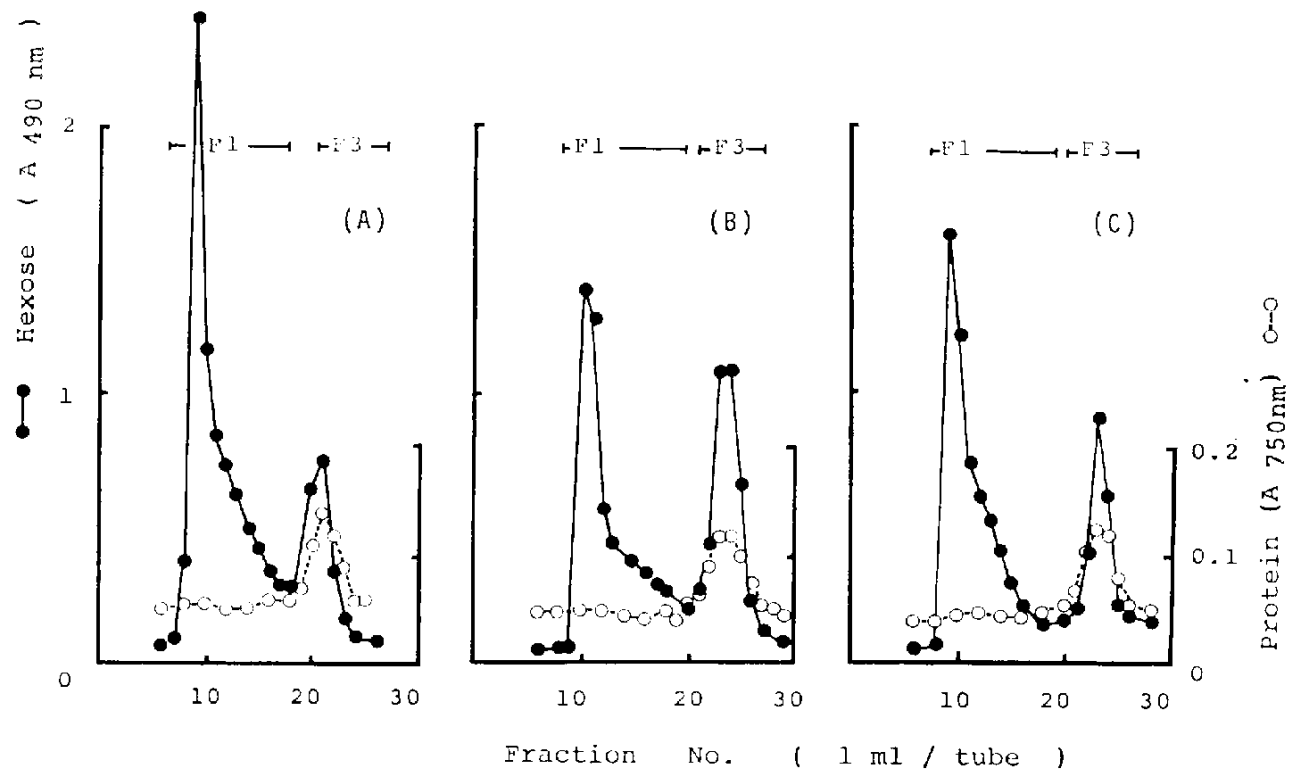

Fig. 6. Gel filtration of the gastric juice on the Bio-Gel A $1.5 \mathrm{~m}$ column. (A) Normal, (B) Control (Aspirin). (C) GGA (300 mg/kg). 


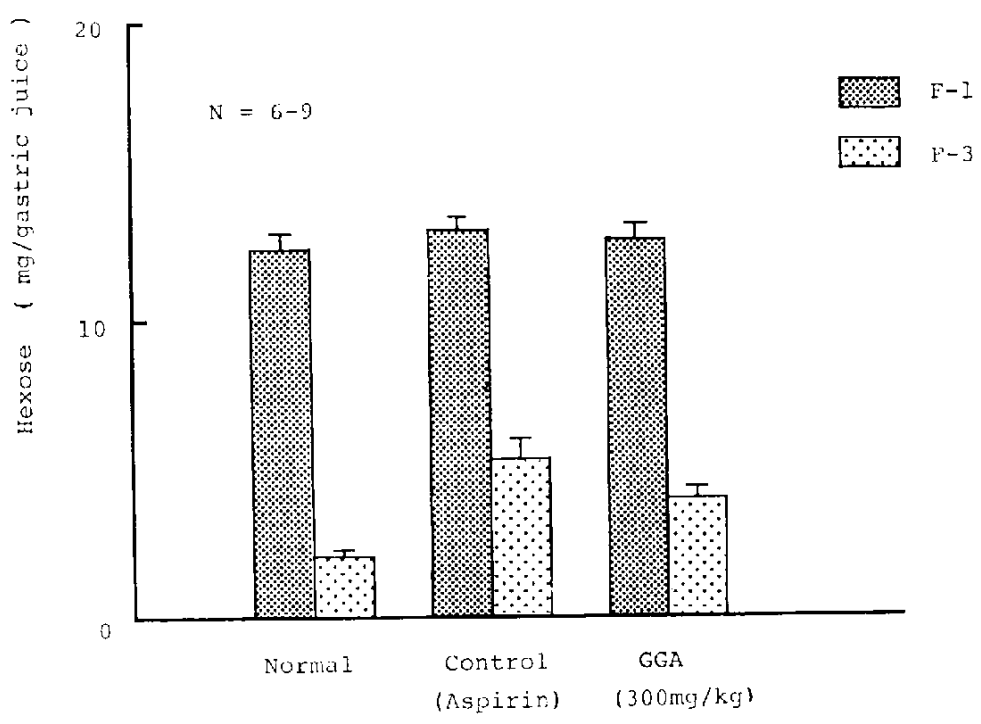

Fig. 7. Changes of the hexose content in the F-1 and F-3 fractions in the gastric juice.

materials ( $F-1)$, which formed a sharp peak at the void volume and broad shoulder, had no detectable protein component, while the smaller materials $(F-3)$ formed one peak which included proteins. The elution position of this former peak, containing high percentage of hexose, corresponded to that of the gastric glycoproteins $\mathrm{F}-1$ and $\mathrm{F}-2$ in the stomach. The latter peak corresponded to $\mathrm{F}-3$ in the stomach. The elution profile of gastric juice in aspirin treated rats (Control) showed that the F-1 peak was smaller and the F-3 peak was larger as compared to those of normal rats (Fig. 6B). In aspirin plus GGA treated rats, the elution pattern was not changed to that of the control rats (Fig. 6C). Each fraction of F-1 and F-3 was pooled, and hexose contents in these fractions were determined. Since the volume of gastric juice was increased in aspirin treated rats $(8.2 \pm 0.7$ to $9.8 \pm 0.7 \mathrm{ml})$, the total amount of $\mathrm{F}-3$ ( $\mu \mathrm{g} / \mathrm{gastric}$ juice) was significantly increased, while that of $F-1$ was not changed to the normal level. GGA showed no significant effect on this change in the gastric juice (Fig. 7).

\section{Discussion}

The present study indicated that the gastric macromolecular glycoproteins were contained in the superficial mucous layer as a highly concentrated state and that the glycoprotein contents were decreased in the mucous layer and gastric mucosa following oral administration of aspirin. GGA, which had been shown to prevent aspirin-induced ulceration (4), could inhibit the decreases in the amount of gastric glycoproteins in the mucous layer and gastric mucosa. These findings were well associated with the changes in PAS positive substances in both layers as examined by the histochemical method. Based on these results, we hypothesized that the gastric macromolecular glycoproteins, which are the mucin type (3). play a role as a defensive mechanism. forming the mucous layer on the surface of the gastric mucosa. Allen et al. (10) have reported that the macromolecular glycoproteins in the human gastric mucosa were dissociated into four subunits by pepsin digestion, and they also discussed the 
protective action of the glycoproteins.

It is unlikely that the decreases in the gastric glycoprotein contents by aspirin are only a result of the mucosal damages since Azuumi et al. (3) demonstrated that the changes in contents of the glycoproteins were detected before the macroscopical changes of the mucosa. The inhibitory effect of aspirin on mucus biosynthesis $(1,2,11)$ or mucus secretion (12) may cause the decreases in the gastric glycoproteins.

Mucus secretion in the gastric juice was also examined by using the same method of gel filtration. The content of the macromolecular fraction in the gastric juice, which had no detectable protein component, was not affected by aspirin. while that of the lower molecular fraction in the gastric juice was significantly increased when compared to the normal level. GGA had no significant effect on this change. It is not clear whether these fractions in the gastric juice are the component of gastric glycoproteins actively released or the degraded materials of gastric glycoproteins by pepsin digestion. Further investigation is necessary to clarify the significance of these changes in the gastric juice.

As previously reported (4), GGA showed a preventive effect on acid-back diffusion induced by aspirin: GGA inhibited the reduction of $\mathrm{H}^{+}$ion concentration and the increment of $\mathrm{Na}^{+}$ion concentration in the gastric juice induced by aspirin. These results suggest the possibility of a correlation between acid-back diffusion and the decreases in gastric glycoproteins in the stomach.

The preventive effect of GGA on the decreases in gastric glycoproteins may be attributed to action on the glycoprotein biosynthesis in the gastric mucosa since it was suggested that GGA might be involved in the glycoprotein synthesis through the enhancement of glycolipid biosynthesis in rat liver microsomes (13).

We have previously shown that GGA could inhibit the decreases in hexosamine content in the stomach induced by aspirin (4) or cold-restraint stress (14). Although hexosamine is contained not only in mucus glycoproteins but also in connective tissues. it was indicated from the present study that the greater part of the hexosamine content in the stomach extract was derived from the gastric macromolecular glycoproteins and that hexosamine content in the cell debris and/or connective tissue was negligible to the total content of hexosamine. These results indicated that GGA might also have a preventive effect on the reduction in gastric glycoproteins by stress and that hexosamine content in the stomach can serve as an indicator of the content of gastric glycoproteins.

In conclusion, the disturbance of biosynthesis of the gastric macromolecular glycoproteins may contribute to ulcer formation by aspirin. GGA showed a preventive effect on the reduction of gastric glycoproteins.

Acknowledgements: The authors are grateful to Mr. M. Ito for his valuable advice and to Dr. K. Ishihara and Dr. Y. Azuumi. Kitasato University School of Medicine. for their comments on the manuscript.

\section{References}

1) Rainsford, K.D.: The effect of aspirin and other non-steroid anti-inflammatory/analgesic drugs on gastro-intestinal mucus glycoprotein biosynthesis in vitro: relationships to ulcerogenic actions. Biochem. Pharmacol. 27, 877-885 (1978)

2) Dekanski, J.B., MacDonald A. and Sacra, P.: Effect of fasting, stress and drugs on gastric glycoprotein synthesis in the rat. Br. J. Pharmacol. 55, 387-392 (1975)

3) Azuumi, Y., Ohara, S., Ishihara, K., Okabe, H. and Hotta, K.: Correlation of quantitative changes of gastric mucosal glycoproteins with aspirin-induced gastric damage in rats. Gut 21 . 533-536 (1980) 
4) Murakami, M. Oketani, K., Fujisaki, H., Wakabayashi, T., Ohgo, T. and Okabe, S.: Effect of antiulcer drug. geranylgeranylacetone on aspirin-induced gastric ulcers in rats. Japan. J. Pharmacol. 32, 299-306 (1982)

5) Okabe, S., Takeuchi, K, Nakamura, K. and Takagi, K.: Pathogenesis of gastric lesioris induced by aspirin in the pylorus-ligated rat. Japan. J. Pharmacol. 24. 363-371 (1974)

6) Murakami, M., Oketani, K., Fujisaki, H., Wakabayashi, T., Ohgo, T. and Kawai, K.: Fundamental studies of the changes of gastric mucosubstances induced by cold-restraint stress in rats. J. Kyoto Pref. Univ. Med. 90, 443-451 (1981)

7) Dubois, M., Gilles, K.A., Hamilton, J.K., Rebers, P.A. and Smith, F.: Colorimetric method for determination of sugars and related substances. Anal. Chem. 28, 350-356 (1956)

8) Wang, C. and Smith, R.L.: Lowry determination of protein in the presence of Triton X-100. Anal. Biochem. 63, 414-417 (1975)

9) Neuhaus, O.W. and Letzring. M: Determination of hexosamine in conjugation with electro- phoresis on starch. Anal. Chem. 29, 1230-1233 (1957)

10) Allen, A. and Garner, A.: Mucus and bicarbonate secretion in the stomach and their role in mucosal protection. Gut 21, 249-261 (1980)

11) Sander, L.D. and Johnson, L.R.: Inhibition of gastrointestinal and liver hexosamine synthesis by sodium salicylate and ethanol. Proc. Soc. Exp. Biol. Med. 165, 424-430 (1980)

12) Menguy, R. and Masters, Y.F.: Effects of aspirin on gastric mucous secretion. Surg. Gynecol. Obstet. 120,92-98 (1965)

13) Murakami, M., Muto, Y., Moriwaki, H. and Ohgo, T.: Effect of acyclic polyisoprenoids on the biosynthesis of mannose-labeled glycolipids in rat liver microsomes. Biochem. Biophys. Res. Commun. 103, 706-712 (1981)

14) Murakami, M., Oketani, K., Fujisaki, H., Wakabayashi, T., Ohgo, T. and Kawai, K.: Effect of geranylgeranylacetone on the changes of gastric mucosubstances in rats subjected to cold-restraint stress. J. Kyoto Pref, Univ. Med. $90,453-459(1981)$ 